\title{
Demonstrate, Observe, Assist, and Perform versus structured educational video in imparting standard skill in male urinary bladder catheterization
}

\author{
Santosh Balakrishnan', Lijo Paul' ${ }^{2}$, Minu N Rajan ${ }^{3}$, Sherin A Arthungal ${ }^{4}$ \\ ${ }^{1}$ Associate Professor, ${ }^{2}$ Senior Resident, 3,4 Junior Resident, Department of Surgery, MOSC Medical College Hospital, \\ Ernakulam, Kerala, India
}

Background: Conventional teaching-learning methods often fail to assure competency in male bladder catheterization (MBC) in the Indian Medical Graduate. Introduction of structured training methods could offer a solution. Aims and Objectives: The objective of the study was to compare efficiency of demonstrate, observe, assist, and perform (DOAP) method of training in a skill lab against structured educational video (SEV)-based learning in training final MBBS students in the psychomotor skill to safely perform MBC. Materials and Methods: Following IRB and IEC clearance, final MBBS students fulfilling selection criteria were randomly allocated into two comparable groups. One group underwent skill lab training using DOAP method while the other group underwent training using a SEV by the same instructor. CRRI interns, regularly performing MBC at work by virtue of conventional training, with 6-8 months experience formed a control group. All participants underwent assessment of skill in MBC by skill lab OSCE evaluation, by assessors blinded to the participant's method of training. Data were recorded and analyzed using standard statistical software. Trial evaluation from the trial groups was obtained using SurveyMonkey tool. Results: There was no statistically significant difference in the ability of DOAP group or SEV group to safely perform MBC though a higher level of confidence was expressed with their training by DOAP group. Both trial groups statistically outperformed the control group. Conclusion: Structured training assures competence. Video-assisted training produces comparable results though DOAP method is preferred by students. A combination of the techniques may facilitate optimal training.

Key words: Competency-based education; Medical education; Simulation

\section{Access this article online}

\section{Website:}

http://nepjol.info/index.php/AJMS DOI: 10.3126/ajms.v13i2.40184

E-ISSN: 2091-0576

P-ISSN: 2467-9100

Copyright (c) 2022 Asian Journal of Medical Sciences

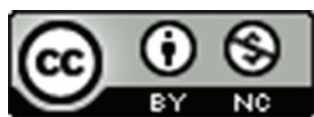

This work is licensed under a Creative Commons Attribution-NonCommercial 4.0 International License.

\section{INTRODUCTION}

Wide variation is observed in skills in performing common bedside clinical procedure of male bladder catheterization among final phase MBBS students trained through conventional training and assessment. ${ }^{1,2}$ Conventional teaching and learning methods may not allow assurance of achievement of competencies in the Indian Medical Graduate. ${ }^{1}$ Introduction of structured training methods could offer a reliable method of facilitation of learning and assured skill acquisition. ${ }^{3,4}$

\section{Aims and objectives}

The team aimed to investigate the suitability of demonstrate, observe, assist, and perform (DOAP) method of training in a skill lab simulator compared with demonstration using a structured educational video (SEV) in imparting psychomotor skill training in a bedside procedure to medical students.

The objective of this trial was to evaluate the efficacy of training by DOAP in comparison to training through a SEV in acquiring skill in performing male bladder catheterization among final phase MBBS students. 


\section{MATERIALS AND METHODS}

The study was designed as a randomized comparative study conducted in the investigator's institution. IRB and IEC clearance were obtained. A SEV was created mirroring the steps and instructions conveyed by the DOAP training session. This SEV was approved through peer-review by four consultant surgeons, one of whom was a consultant urologist. The DOAP session and OSCE assessment of all groups were conducted in a simulation laboratory with procedure demonstrated and assessed using a standard male catheterization trainer model. The group being trained through a SEV was trained by being shown the video twice in the seminar room in the presence of the trainer who led the training session and also reinforced the steps by verbal repetition between two successive viewings of the SEV.

The larger population of the study are final phase MBBS Students of Ernakulam district in Kerala, India.

\section{Sampling method}

All final phase MBBS students fulfilling selection criteria were randomly assigned a trial number by blinded chit pickup for anonymity with a securely held key for verification if necessary. They were divided into two equal groups based on random selection and grouping again by blinded chit pick-ups. Compulsory Residential Rotating Internship (CRRI) interns were recruited through their random allocation to the department as per their rotation, to serve as a control group. The interns posted to the department for the duration of the study would have completed between 6 and 8 months of CRRI with regular opportunity at work to perform male bladder catheterization (MBC). They represent a comparable group who have just completed final phase MBBS and have learned the procedure through the conventional apprenticeship method.

\section{Inclusion criteria}

The following criteria were included in the study:

1. Medical students from final phase who have not had skill training in male bladder catheterization who consent to participate.

2. CRRI interns posted to the department of surgery subject to their consent.

\section{Exclusion criteria}

The following criteria were excluded from the study:

1. Medical students who have had structured skill training through any means in bladder catheterization.

2. CRRI interns who had any structured training in bladder catheterization other than traditional apprenticeship.

3. CRRI interns from the senior additional batch.

4. Subjects who have allergy to latex.

\section{Sample size}

All final phase MBBS students who fulfill inclusion criteria were recruited. The number of participants was limited by the maximum number of students fulfilling criteria in the institution over the permitted study period.

\section{Intervention}

Institutional Review Board and Ethical Committee clearance and written informed consent from all participants were taken. The DOAP group underwent structured training using the DOAP method in batches of not more than 6 subjects with demonstration and observation as a group and individual opportunity to assist and perform. The group being trained through a SEV was trained in batches of 15 , as limited by the size of the seminar room available for screening the video. They were shown the video 2 times in the presence of the trainer who verbally reinforced the steps between viewings, to match the visual and auditory exposure through DOAP. A group of 35 CRRI interns posted in our department in numbers to reasonably match the test groups, act as controls trained by conventional apprenticeship method of training to verify the validity, and impact of either of the study methods of training over current practice as recommended by members of the institutional research committee. Participants in all groups were individually assessed and scored using an existing validated OSCE assessment form ${ }^{5}$ for their skill in male catheterization by assessors blinded to the intervention in question. The OSCE form assessed completion of 26 vital steps of the procedure. Eight vital steps were allocated two marks while all others had one mark making up a total score of 34. Following assessments, an online program satisfaction evaluation questionnaire was administered to each participant. Debrief to subjects was provided only after collection of evaluation questionnaire. In the interest of fairness, a crossover training was provided by sending the video to all in the DOAP group and DOAP training was offered to all in the SEV group after the assessments and online evaluation was complete to avoid bias in response.

\section{Data collection method and analysis}

OSCE valuation scores in whole numbers for all groups were compiled in Excel sheets. SPSS software was used for data analysis. The institutional bio-statistician aided the analysis in view of the authors' limited skill with the software tool. $\mathrm{P}<0.05$ was considered as statistically significant. OSCE scores were summarized using appropriate tests of significance using these tools. Evaluation response received from both groups using SurveyMonkey online survey tool, was analyzed for difference in levels of agreement on various elements of the training experience with their allocated training method after they completed the OSCE assessment. Data were analyzed using MS Excel. 


\section{RESULTS}

All trial intervention groups; DOAP group ( $\mathrm{n}=39)$, SEV group ( $\mathrm{n}=39)$, and the control group participants $(\mathrm{n}=35)$ successfully completed catheterization in the Simulator model in the skills lab. OSCE scores were awarded by assessors blinded to the interventions. Weight given to each of the 26 elements in the OSCE assessment as well as total scores out of 34 were recorded on pro forma and compiled on MS Excel. The scores were found to follow normal distribution (Kolmogorov-Smirnov test) in control, DOAP, and SEV groups (Figure 1).

Means and standard deviations were calculated. Initial oneway ANOVA was performed using SPSS software to assess significant difference in the mean scores for each of the three groups. Fisher's exact test showed $(\mathrm{F}$ value $=65.53$; $\mathrm{P}<0.001)$ significant difference between the three groups.

In keeping with the objective of the study, a post hoc test (Tukey-HSD) performed to compare the DOAP and SEV groups was done. There was no significant difference between the mean scores of the two groups $(\mathrm{P}=0.92)$. There was significant difference, however, between SEV and DOAP groups individually against the control group (Table 1).

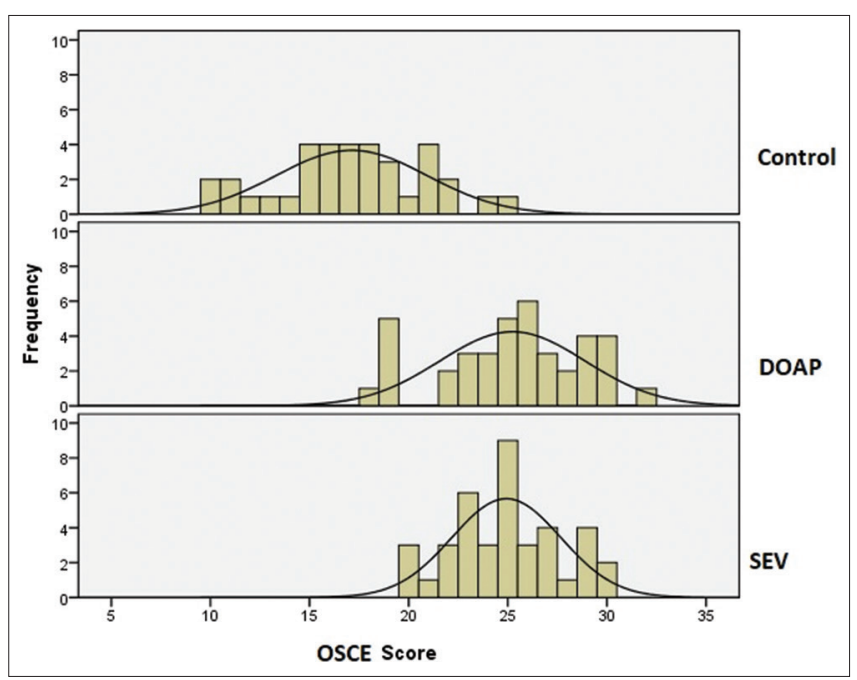

Figure 1: Histogram of OSCE results: Normal distribution
Assessment of the feedback response on SurveyMonkey showed a statistically significant difference in the level of agreement with all elements of training surveyed favoring the DOAP group (Table 2).

In a subanalysis, eight critical elements of the procedure assessed in the OSCE (Questions 3, 6, 10, 14, 17, 18, 22, and 26) were compiled (Table 3).

Failure to perform each critical element of the procedure was compared between DOAP and SEV groups. It was observed that contrary to the respondent's feedback, the SEV group actually outperformed the DOAP group in the elements of the OSCE $(\mathrm{Q} 3,10,17$, and 18) that pertained to safety and risk mitigation in the procedure. They performed poorly in the steps (Q6, 14, 22, and 26) that pertained to pre-procedure hand wash, adequate use of lubricant anesthetic gel, post-procedure replacement of prepuce to normal position, and documentation in the notes. The difference failed to achieve statistical significance in any except Q22, pertaining to repositioning of prepuce post-procedure, which favored of the DOAP group.

Despite the significant lower level of confidence expressed by the SEV group, there was no statistically significant difference in their ability to perform the procedure safely vis-à-vis the DOAP group as seen by their OSCE score assessment.

Each of the intervention groups outperformed the control group with the difference reaching statistical significance.

\section{DISCUSSION}

The training of a medical graduate requires development of almost every domain of learning. Most core competencies require the application of more than 1 skill domain. The move toward competency-based medical education (CBME) globally had highlighted this need and also the challenges inherent to ensuring achievement of these competencies. ${ }^{1}$ Wide variability in procedural skills has been observed among junior doctors even after adoption of CBME raising questions about their readiness to undertake roles expected of them. ${ }^{2}$

\section{Table 1: ANOVA comparison of groups}

\begin{tabular}{|c|c|c|c|c|c|c|c|c|}
\hline \multicolumn{3}{|c|}{ OSCE score results } & \multicolumn{2}{|c|}{ Three-way ANOVA } & \multicolumn{4}{|c|}{$\begin{array}{c}\text { One-way ANOVA with Tukey HSD post hoc intergroup } \\
\text { comparison }\end{array}$} \\
\hline Group & n=Numbers & $\begin{array}{l}\text { Mean OSCE } \\
\text { score }\end{array}$ & F Value & P-value & $\begin{array}{l}\text { Comparison of } \\
\text { groups }\end{array}$ & $\begin{array}{l}\text { Mean } \\
\text { difference }\end{array}$ & $P$ value & Significant favoring \\
\hline Control & 35 & 17.11 & 65.528 & $<0.001$ & DOAP vs. SEV & 0.308 & 0.917 & Neither \\
\hline DOAP & 39 & 25.23 & & & Control vs. DOAP & -8.116 & $<0.001$ & DOAP \\
\hline SEV & 39 & 24.92 & & & Control vs. SEV & -7.809 & $<0.001$ & SEV \\
\hline
\end{tabular}

DOAP: Demonstrate, observe, assist, and perform, SEV: Structured educational video, vs.: Versus 


\begin{tabular}{|c|c|c|c|c|}
\hline Questions presented & Groups & $\begin{array}{c}\text { Numbers in } \\
\text { strong agreement }\end{array}$ & $\begin{array}{c}\text { Percentage in } \\
\text { strong agreement }\end{array}$ & $\begin{array}{c}P \text {-value: Significant } \\
\text { if } P<0.05\end{array}$ \\
\hline \multirow[t]{2}{*}{ 1. Captivating and promotes interest } & DOAP & 25 & 73.5 & 0.002 \\
\hline & SEV & 12 & 34.3 & \\
\hline \multirow[t]{2}{*}{ 2. Clear explanation of procedure } & DOAP & 25 & 73.5 & 0.007 \\
\hline & SEV & 14 & 40 & \\
\hline \multirow{2}{*}{ 3. Elements of risk and safety conveyed } & DOAP & 18 & 52.9 & 0.045 \\
\hline & SEV & 9 & 25.7 & \\
\hline \multirow{2}{*}{ 4. Confidence afforded to perform } & DOAP & 17 & 50 & $<0.001$ \\
\hline & SEV & 4 & 11.4 & \\
\hline \multirow[t]{2}{*}{ 5. Suitability of teaching method } & DOAP & 26 & 76.5 & $<0.001$ \\
\hline & SEV & 5 & 14.3 & \\
\hline \multirow[t]{2}{*}{ 6. Whether they would recommend this method } & DOAP & 25 & 73.5 & $<0.001$ \\
\hline & SEV & 3 & 8.6 & \\
\hline \multirow[t]{2}{*}{ 7. Overall satisfaction with teaching method } & DOAP & 26 & 76.5 & $<0.001$ \\
\hline & SEV & 7 & 20 & \\
\hline
\end{tabular}

Degree of affirmative agreement with the teaching method was significantly higher in the DOAP group with $P<0.05$ in every element. DOAP: Demonstrate, observe, assist, and perform, SEV: Structured educational video

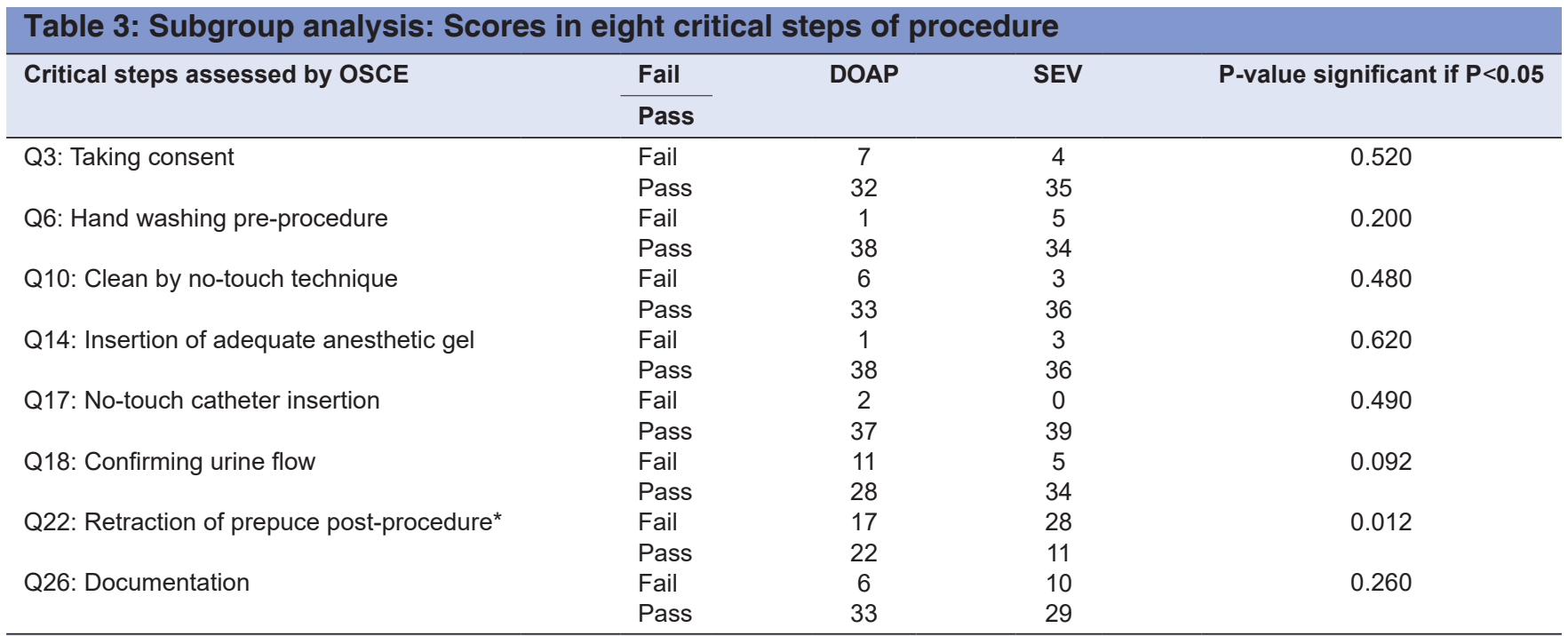

*Bias correction. Q22 discounted because MBC simulator had a circumcised penile shaft and hence could not be reliably assessed on OSCE. DOAP: Demonstrate, observe, assist, and perform, SEV: Structured educational video

Simulation-based training provides an evidence-based solution to imparting skills to the modern trainee in the current practice backdrop of reduced availability of trainee patient contact time as a result of reduced inpatient stay for most treatments and reduced tolerance for trainingrelated morbidity. ${ }^{3}$ Skill labs allow repeated training and assessment in a safe environment till achievement of skills in objectively demonstrated before advancing to the clinical settings. Peyton's 4-step technique (DOAP) is an effective method of psychomotor skill training. This technique has been shown to be significantly effective in procedures involving multiple sequential steps. ${ }^{4,6}$

Many studies have confirmed the positive significant benefit of adding multimedia-based training to traditional text-based training. ${ }^{7}$ Students have also shown greater satisfaction with the use of video-assisted learning in comparison with traditional methods of skill training through demonstrations. ${ }^{8}$ No significant difference was noted in procedural skill when video-based teaching was compared to live demonstrations for training in orthodontic procedures. ${ }^{8}$ Students report personal comfort, availability of media for review and clarification and scope for personal visualization and reflection as possible reasons for this preference. ${ }^{8,9}$

The present study showed that though the subjects trained through DOAP reported greater satisfaction and confidence with their training as compared to those trained through the use of a SEV, there was no significant difference in their OSCE performance scores in the skill lab. There was no statistical difference even in the analysis of eight critical steps selected from the OSCE questionnaire apart from the step requiring the 
subject to declare the need to protract the prepuce post-catheterization in uncircumcised individuals where the difference reached statistical significance favoring the DOAP group. The DOAP group also had a numerical advantage over the SEV group in performing documentation of the procedure, though the difference did not reach statistical significance.

This marginal advantage could be accounted to the simulator model featuring a circumcised penis and the need to document the procedure being conveyed only as a closing statement by the narrator in the SEV rather than a scene of this being done.

The lack of visual impact of both these steps could have resulted in failure on the part of the subject to register the verbal suggestion impressing the need to protract the prepuce post-catheterization in uncircumcised males and ensure documentation of the procedure in the case notes. Including visuals of these steps being performed in the training video could potentially level the field.

Video-based training thus appears to be as effective a tool when compared to the DOAP method for training in simple bedside clinical procedures.

There is evidence that structured training improves performance in health-care professionals in procedural, communication, and clinical examination skills over learning by apprenticeship and informal workplace-based learning. ${ }^{10,11}$

The present study findings also show a clear statistical advantage in the OSCE scores achieved by subjects trained through either method of structured training (DOAP or SEV) over the subjects in the control group who were trained by the conventional way through apprenticeship and unstructured work-based supervised training.

\section{Limitations of the study}

Inherent bias and redressal:

1. Researcher's interest in structured competency-based training:

- Excluded by recruiting two trial blinded assessors for each group assessment.

2. Repetition bias for DOAP group:

- Minimized by limiting feedback to after completing OSCE assessment and submitting evaluation questionnaire.

- SEV group shown video 2 times in presence of trainer to match visual impact with opportunity to discuss steps with trainer to match opportunity afforded to DOAP group.

\section{CONCLUSION}

Based on the analysis of results, we conclude that structured training appears to be a key element in implementing CBME to ensure development of competencies. Personalized training through DOAP in small groups appears to be the ideal method of imparting training in common bedside clinical procedures but needs more time and faculty hours. Training using structured educational videos with the presence and reinforcement by a trainer allows training of larger groups and appears to be equally effective.

We hypothesize that a combination of the techniques could facilitate training through optimal use of faculty and simulation laboratory resource without compromising learning. This hypothesis will need verification and could form the basis of a future trial.

\section{ACKNOWLEDGMENT}

1. Administration of MOSC Medical College; Dean, Dr. Divaker KK. Medical Superintendent, Dr. Sojan Ipe for permitting use of premises and skill lab for the study.

2. Faculty of the National Medical Council nodal center, Kottayam, and my peers from the advanced course of Medical Education (ACME Batch 2019B).

3. My colleagues: Dr. Vergis, Dr. Vijy, Dr. Satish, Dr. Shal and Dr. Koruth, for their support and peer review of the SEV.

4. Institutional Bio-Statistician: Mr. Kalesh M Karun for his support with statistical analysis.

5. Our interns: Batch of 2014 Our Students: Batch of 2015; Skill Lab nurse: Mr. Yaldho, Department Clerk: Ms. Sini. Sister In-charge OT: Ms. Anuja.

\section{REFERENCES}

1. Vogel D and Harendza S. Basic practical skills teaching and learning in undergraduate medical education-a review on methodological evidence. GMS J Med Educ. 2016;33(4):Doc64. https://doi.org/10.3205/zma001063

2. Bugaj TJ, Nikendei C, Groener JB, Stiepak J, Huber J, Möltner A, et al. Ready to run the wards?-A descriptive follow-up study assessing future doctors' clinical skills. BMC Med Educ. 2018;18(1):257.

https://doi.org/10.1186/s12909-018-1370-4

3. Scalese RJ, Obeso VT and Issenberg SB. Simulation technology for skills training and competency assessment in medical education. J Gen Intern Med. 2008;23 Suppl 1:46-49. https://doi.org/10.1007/s11606-007-0283-4

4. Balafoutas D, Joukhadar R, Kiesel M, Häusler S, Loeb S, Woeckel $A$, et al. The role of deconstructive teaching in the training of laparoscopy. JSLS.2019;23(2):e2019.00020. https://doi.org/10.4293/JSLS.2019.00020 
5. University of California Irvine School of Medicine Medical Education Simulation Center; 2015. Available from: http://www. sites.uci.edu/medsim/files/2015/04/foley-catheter-insertionchecklist.pdf

6. Gradl-Dietsch G, Lübke C, Horst K, Simon M, Modabber A, Sönmez TT, et al. Peyton's four-step approach for teaching complex spinal manipulation techniques-a prospective randomized trial. BMC Med Educ. 2016;16(1):284.

https://doi.org/10.1186\%2Fs12909-016-0804-0

7. Buch SV, Treschow FP, Svendsen JB and Worm BS. Videoor text-based e-learning when teaching clinical procedures? A randomized controlled trial. Adv Med Educ Pract. 2014;5:257-262.

https://doi.org/10.2147\%2famep.s62473

8. Botelho MG, Gao X and Jagannathan N. A qualitative analysis of students' perceptions of videos to support learning in a psychomotor skills course. Eur J Dent Educ. 2019;23(1):20-27. https://doi.org/10.1111/eje.12373

9. Alqahtani ND, Al-Jewair T, Al-Moammar K, Albarakati SF and ALkofide EA. Live demonstration versus procedural video: A comparison of two methods for teaching an orthodontic laboratory procedure. BMC Med Educ. 2015;15:199. https://doi.org/10.1186\%2Fs12909-015-0479-y

10. Jünger J, Schäfer $S$, Roth $C$, Schellberg $D$, Ben-David MF and Nikendei C. Effects of basic clinical skills training on objective structured clinical examination performance. Med Educ. 2005;39(10):1015-1020.

https://doi.org/10.1111/j.1365-2929.2005.02266.x

11. Rebuck D, Dzyngel B, Khan K, Kesten RN and Chapman KR. The effect of structured versus conventional inhaler education in medical housestaff. J Asthma. 1996;33(6):385-393. https://doi.org/10.3109/02770909609068183

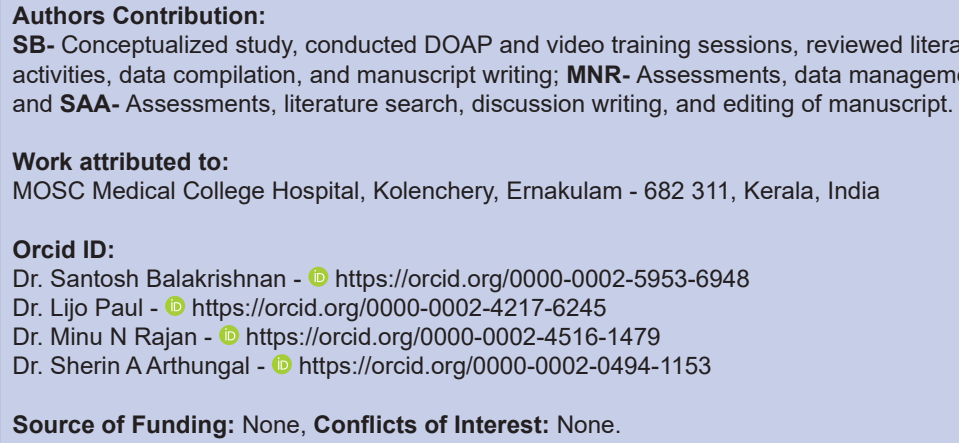

